\title{
GESTIÓN TECNOLÓGICA COMO FACTOR CLAVE DE ÉXITO EN UNIVERSIDADES PRIVADAS*
}

Technology management as factor key to success in private universities

\author{
Reynier Israel Ramírez Molina**; Giovanni Antonio Royero Orozco*** \\ y El Kadi Janbieh, Omar Nabih****
}

\section{RESUMEN}

La investigación tuvo como propósito describir la gestión tecnológica como factor clave de éxito en las universidades privadas del municipio Maracaibo. El tipo de investigación fue descriptiva, de campo y transaccional, no experimental. La población estuvo conformada por (4) universidades privadas ubicadas en el municipio Maracaibo, con un total de cincuenta y cinco (55) unidades informantes integradas por jefes de servicios web, redes, servidores, servicios técnicos, plataformas operacionales y operadores técnicos. Para la recolección de datos se aplicó un cuestionario estructurado, constituido por doce (12) ítems de cinco (5) alternativas de respuestas

\footnotetext{
* Este capítulo deriva del trabajo de investigación intitulado: "Procesos de la gestión tecnológica como factor clave de éxito en las universidades privadas del municipio Maracaibo", en el marco del Programa Postdoctoral sobre Gerencia de las Organizaciones, impartido en la Universidad Privada Dr. "Rafael Belloso Chacín".

**Postdoctor en Gerencia en las Organizaciones. Doctor en Ciencias de la Educación. Magíster Scientiarum en Gerencia de Recursos Humanos. Licenciado en Comunicación Social, Mención Publicidad y RR.PP. Docente Investigador, tiempo completo en la Universidad de la Costa, Barranquilla, Colombia. Director Administrativo de la Fundación Villa Bernarda (FUNDAVIBE), Maracaibo, Venezuela. Correo electrónico: rramirez13@cuc.edu.coORCID ID: http://orcid.org/0000-0002-5073-5158.

***Postdoctor en Gerencia en las Organizaciones. Doctor en Ciencias Gerenciales. Magíster Scientiarum en Ingeniería Geodésica. Ingeniero Geodesta. Docente Investigador, dedicación exclusiva en la Universidad del Zulia-LUZ, Maracaibo - Zulia, Venezuela. Miembro de la Sociedad Civil de Especialistas Latinoamericanos en Percepción Remota y Sistemas de Información Espacial (SELPER) Capítulo Venezuela y Coordinador de la Región Zulia. Miembro del Grupo de Investigación del Laboratorio de Geodesia Física y Satelital (LGFS) de la Universidad del Zulia. Director de la División de Investigación Facultad de Ingeniería, Universidad del Zulia, Miembro de la Comisión Científica del Consejo de Desarrollo Científico y Humanístico de la Universidad del Zulia (CONDES-LUZ). Maracaibo, Venezuela. Correo electrónico: groyero@fing.luz.edu.ve

${ }^{* * * *}$ Postdoctor en Gestión de la Ciencia y la Tecnología. Postdoctor en Integración y Desarrollo de América Latina. Postdoctor en Gerencia Pública y Gobierno. Postdoctor en Gerencia en las Organizaciones. Doctor en Ciencias Gerenciales. MBA en Finanzas y Mercado Internacional. Ingeniero de Computación. Coordinador de la Maestría de Gerencia de Mercadeo y Docente Investigador, dedicación exclusiva en URBE, Maracaibo, Venezuela. Correo electrónico:oelkadi@urbe.edu.veORCID
} ID: https://orcid.org/0000-0002-5976-932X 
con escala de tipo Likert, la validez del instrumento se determinó a través de diez (10) expertos. La confiabilidad se estableció a través del cálculo de coeficiente de alfa cronbach obteniendo un valor de 0.80r, siendo este altamente confiable. De acuerdo a los resultados obtenidos, se percibe un mediano cumplimiento en la aplicabilidad de las actividades de investigación y desarrollo, innovación tecnológica, producción y administración estratégica, y utilidad adecuada de la tecnología por el capital social. Encaminados a fortalecer el desarrollo investigativo, originando una comunidad de práctica, aprendizaje, saber, conocimiento y trasformación, que utilizan adecuadamente los recursos tecnológicos para tal fin, posibilitando la construcción y permanencia de los grupos de investigación, centros de desarrollo tecnológicos y en redes. En síntesis, los datos presentados permiten demostrar que la gestión tecnológica es un factor clave de éxito en las universidades privadas, vinculando el sector productivo y de la investigación/desarrollo, en el proceso de innovación tecnológica, originando conocimientos y prácticas relacionadas con la creación, desarrollo, transferencia y uso de la tecnología.

Palabras clave: gestión tecnológica, investigación y desarrollo, innovación tecnológica, producción y administración estratégica, uso tecnológico.

RECIBIDO: Febrero 2018

\section{ACEPTADO: Septiembre 2018}

\section{ABSTRACT}

The research purpose was to describe the technology management as a key factor of success in the private universities in the municipality Maracaibo. The research was descriptive, field and transactional, non-experimental. The population was comprised of (4) private universities located in the Maracaibo municipality, with a total of fiftyfive (55) reporting units composed of Heads of web services, networks, servers, technical services, operational platforms and technical operators. A questionnaire structured, consisting of twelve (12) items from five (5) alternatives to answers with Likert-type scale was applied to data collection, the validity of the instrument is determined through ten (10) experts. Reliability is established by calculating cronbach's coefficient Alpha obtaining a value of 0.80 r, being this highly reliable. According to results, perceived a medium compliance in the applicability of the activities of research and development, technological innovation, production and strategic management, and proper utility of the technology by the capital social.Aimed at strengthening the development research, giving rise to a community of practice, learning, knowing, knowledge and transformation, properly using the technological 
resources to do so, allowing the construction and permanence of the research groups, centers of development technology and networks. In summary, presented data to show that the technology management is a key factor of success in the private universities, linking the sector production and research/development, in the process of technological innovation, originating knowledge and practices related to the creation, development, transfer and use of technology.

Key words: technology management, research and development, technological innovation, production and strategic management, technology use.

\section{Introducción}

A lo largo del siglo XXI las organizaciones han ido evolucionando a la par de la tecnología. El hombre se ha ocupado en generar nuevas maneras de operar los equipos y alcanzar mejores desarrollos tecnológicos, logrando objetivos organizacionales de una manera eficaz, segura y que garantice la creación de productos o servicios acordes a las necesidades de los usuarios.

Así mismo, dar paso a un entorno local de una economía globalizada, trae consigo modificaciones en los mercados, formas de negociación, valores sociales, políticas de ciencia, tecnología e innovación, entre otros. Estos cambios se han acelerado con el avance de las tecnologías, la transición del perfil productivo de los bienes a los servicios en la gestión tecnológica se ha visto en las organizaciones como aspectos claves, garantizando una sostenibilidad, orientado por el desarrollo e innovación, donde el talento humano juego un rol importante en las organizaciones.

El desarrollo de políticas de ciencia, tecnología e innovación acertadas, representa uno de los instrumentos fundamentales para promover la productividad, competitividad y desarrollo socioeconómico del mundo. Desde esta visión, es esencial la elaboración de políticas que fortalezcan las capacidades científico - tecnológicas de la nación, eleven los niveles de progreso, así como de bienestar social de toda la población, (Aguilar, 2017).

La gestión tecnológica se ha presentado en las organizaciones, como una herramienta cargada tanto de teorías como prácticas, permitiendo a los líderes, 
Reynier Ramírez; Giovanni Royero y Omar El Kadi

Telos Vol. 21, No. 1 (2019). 10-32

desplegar estrategias congruentes con sus planes de negocios. Todo esto se ve reflejado en las estrategias técnicas para la adquisición, uso y creación de tecnología que exige las condiciones del mercado.

De ese modo, la gestión tecnológica se orienta a la integración del proceso de cambio con aspectos estratégicos, operativos y toma de decisiones de la organización, representando un valor competitivo, por tanto, debe formar parte del planteamiento estratégico, vinculando las actividades de investigación y desarrollo (I+D), innovación, producción y administración estratégica, y la utilidad adecuada de la tecnología por el capital social.

Ante tales consideraciones, la gestión tecnológica posibilitará el desarrollo de procesos productivos eficazmente, logrando con ello aumentar las expectativas de sus clientes y como consecuencia, su satisfacción. Como parte de la gestión, las organizaciones deben ejecutar decisiones eficientes, respondiendo al ritmo con el cual las tecnologías entran al mercado, enfrentando los desafíos de los agentes de cambio.

Así mismo, la adquisición de tecnología, establece posibles fuentes de financiación, análisis de costos y viabilidad de sus alcances necesarios para realizar el cambio técnico programado evaluando las alternativas disponibles. Esta, debe comenzar con la realización del inventario tecnológico a través de recopilaciones, que amplía el conocimiento acerca de lo que acontece en el mundo en materia tecnológica. Para tal fin, las empresas deben hacer investigaciones externas aplicando herramientas de investigación, como análisis de mercado, benchmarking o auditorías, para la administración adecuada de los recursos, permitiendo conocer el nivel tecnológico trabajado.

Según Robledo (2013), la innovación desborda el marco empresarial y se desarrolla en un contexto social amplio que involucra una gran diversidad de instituciones, en tal sentido es interesante examinar el ámbito empresarial de la innovación, bajo la consideración de la importancia que la organización tiene en este fenómeno. La innovación exitosa tampoco sería suficientemente significativa en 
términos sociales, sin mecanismos adicionales que garanticen altos niveles de impacto. Tal mecanismo es la difusión como fenómeno y concepto económico, referido a la adopción de la innovación en la gestión tecnológica que hacen de la misma los agentes económicos sociales.

En cuanto a las instituciones universitarias, la gestión tecnología se ve afectada en un mercado demandante de divisas para el mantenimiento de las plataformas tecnológicas, en la adquisición de tecnología, transferencia, innovación, actividades de investigación y desarrollo, así como también la negociación de las tecnologías y capacitación del capital humano, se ha lidiado durante estos años con un control cambiario y un marco regulatorio, que impone normas lo que genera incumplimientos a los compromisos con los proveedores internacionales, para ofrecer servicios de alta calidad al cliente.

En ese sentido el estado, mediante diferentes medidas, ha decidido proteger con divisas preferenciales algunos rubros de los sectores que hacen vida en el país, excluyendo a las universidades privadas dentro de esa prioridad viéndose afectado la tecnología como parte del crecimiento sostenido, afectando la gestión en la toma de decisiones. El sector universitario está teniendo dificultades financieras para dar respuesta al crecimiento sostenido en la mejora dela gestión tecnológica garante en ayudar a los requerimientos del universo pertinente.

De continuar la situación descrita anteriormente el sector se verá limitado drásticamente en la libre elección de adquirir, investigar, desarrollar, innovar, transferir y administrar su tecnología, en el mediano plazo, aumentando la posibilidad de involución, permitiendo la obsolescencia en los procesos y adecuada calidad de los servicios al cliente.

Las causas de estos fenómenos se ven reflejadas por factores políticos económicos, que a su vez producen una fuerte devaluación de la moneda circulante, traducida en una fuerte inflación, originando restricción a la hora de adquirir divisas, lo que ocasionaría el descenso en la competitividad institucional, impactando las 
Reynier Ramírez; Giovanni Royero y Omar El Kadi

Telos Vol. 21, No. 1 (2019). 10-32

actividades de I+D, innovación, producción y administración estratégica, y la utilidad adecuada de la tecnología por el capital social.

Con referencia a lo anterior, se evidencia una posible debilidad en la gestión tecnológica, considerando la innovación, adquisición, negociacióny transferencia de esta. En consecuencia, existe una escasa orientación hacia la promoción mediante el estímulo del desarrollo de las capacidades técnicas - locales, en el sector universitario para alcanzar un desarrollo sostenido en las diferentes áreas de servicio, comercialización, diseño de equipos, estructuras y sistema de apoyo de la organización.

Los planteamientos expuestos, no escapan a la realidad de las universidades privadas del municipio Maracaibo, entre estas la: Universidad Privada Dr. Rafael Belloso Chacín (URBE), Universidad Rafael Urdaneta (URU), Universidad José Gregorio Hernández (UJGH) y la Universidad Cecilio Acosta (UNICA), en las cuales, al parecer, la gestión tecnológica apenas alcanza un estado inicial. Esta apreciación fue observada a través de entrevistas previas realizadas por los investigadores.

Con referencia a lo anterior reviste una importancia significativa, y debe ser atendido de inmediato, pues de seguir bajo ese esquema, el detrimento de las universidades privadas será un hecho, imposibilitando crecer tecnológicamente, mediante las mejoras de los servicios, adquisición de tecnología y alianzas estratégica, pues el mundo avanza a un ritmo acelerado, al igual que el mercado, por lo que la organización deben estar a la vanguardia para permanecer actualizadas, en busca de la competitividad. Ante dicho planteamiento se formula como interrogante: ¿Cómo es la gestión tecnológica como factor clave de éxito en las universidades privadas del municipio Maracaibo?

Por tal motivo, se busca describir la gestión tecnológica como factor clave de éxito en las universidades privadas del municipio Maracaibo. Ayudando a las universidades, a prepararse para el futuro, reduciendo sus riesgos comerciales, incertidumbres del negocio a base de incrementar su flexibilidad, y comprensión de la 
evolución de los productos o servicios del mismo, mediados por actividades de I+D, innovación, producción y administración estratégica, y la utilidad adecuada de la tecnología por el capital social.

\section{Gestión Tecnológica: una revisión conceptual}

Con el propósito de ser competitivas y mantenerse en el mercado, las organizaciones han ido incorporado la gestión tecnológica en sus principios corporativos, asociado a su cadena de valor sistémica, que garantice los recursos disponibles, para el cumplimiento de sus indicadores de logro, como factores claves de éxito. La gestión tecnológica involucra actividades de $\mathrm{I}+\mathrm{D}$, innovación, producción y administración estratégica, y la utilidad adecuada de la tecnología por el capital social, con el propósito de crear valor mediante ventajas diferenciadoras cualificables para enfrentar las exigencias del mercado.

La gestión tecnológica empresarial busca: (a) maximizar sus ventajas competitivas, basadas en su capacidad de desarrollo e innovación tecnológica, y en la obtención y uso sistemático de los medios tecnológicos y organizacionales; (b) congruencia organizacional y método para los esfuerzos de desarrollo tecnológico, de incorporación de tecnologías distintivas e innovación tecnológica, que llevan a cabo en sus procesos de creación, transformación y entrega de valor a clientes y consumidores; y, (c) complementan el esfuerzo que las empresas realizan para agregar valor a sus productos o servicios, (Medellín, 2010).

Para Mendoza y Valenzuela (2014), el rol de la gestión tecnológica, se refiere a la innovación, actividades de investigación para garantizar su práctica e impacto. Por su parte, Hernández, Cardona y Del Rio (2017), exponen que estas se orientan por las actividades de $\mathrm{I}+\mathrm{D}$, e innovación, con el dominio de conocimientos, habilidades mediante destrezas asociados a esos procesos que constituyen las capacidades tecnológicas; posibilitando las actividades involucradas en el desarrollo tecnológico, pero también todo lo que una organización hace en su cadena de valor.

De igual manera Onoyama, Machado, Inoue y Freitas (2017), exponen que la gestión tecnológica, es el conjunto de conocimientos y actividades capaces de generar 
valor a la gestión organizacional mediados por el uso tecnológico, permitiendo una producción y administración estratégica en la ejecución de sus tareas, aumentando la competitividad organizacional en el mercado.

Mientras que para Wu, Liu y Chin (2018), la gestión tecnológica, busca el aumento de la innovación en sus aspectos estratégicos y operativos según la naturaleza de cada organización. Apoyado por la filosofía y objetivos claves, utilizando la tecnología adecuadamente por el capital social, adaptadas a las necesidades del sector productivo.

Tomando como referencia los aportes conceptuales de Medellín (2010), Mendoza y otros, (2014), Hernández y otros (2017), Onoyama y otros (2017) y Wu y otros (2018), posibilita el análisis de las dimensiones que contempla la gestión tecnológica, entre estas las actividades de $\mathrm{I}+\mathrm{D}$, innovación tecnológica, producción y administración estratégica, y la utilidad adecuada de la tecnología por el capital social, impactando transversalmente los resultados de la gerencia universitaria, considerado como factor clave de éxito, (tabla 1).

\section{Tabla 1}

\section{Gestión tecnológica desde la sistematización conceptual}

Gestión tecnológica Sistematización conceptual

Trabajo creativo llevado a cabo de forma sistemática para incrementar el volumen de conocimientos.

Actividades de I+D.

Uso de conocimientos para crear nuevas aplicaciones.

Aplicación del conocimiento existente para desarrollar nuevos productos y procesos.

Desarrollo de tecnologías con impacto sostenible en costo y diferenciación, pudiendo ser replicadas por la competencia.

Creación o modificación de las características de un producto o servicio.

Comunicación para la efectividad de gestión en las organizaciones.

Innovación tecnológica. Búsqueda de alternativas que proporcionen condiciones de uso efectivas.

Mejoramiento de productos, servicios y procesos.

Utilización de habilidades o recursos para desarrollar nuevos bienes/servicios, satisfaciendo las necesidades del usuario. 


\section{Gestión tecnológica como factor clave de éxito en universidades privadas}

Producción y administración estratégica.

Utilidad adecuada de la tecnología por el capital social.
Formulación, ejecución y evaluación de acciones que permiten lograr los objetivos organizacionales.

Uso adecuado de los recursos tecnológicos en productos, servicios, procesos y áreas estructurales-funcionales, para solventar cualquier problema de la sociedad, ser humano y ambiente.

Fuente: elaboración propia.

Las actividades de investigación y desarrollo (I+D): comprenden el trabajo creativo llevado a cabo de forma sistemática para incrementar el volumen de conocimientos, incluido el conocimiento del hombre, cultura - sociedad, y el uso de esos conocimientos para crear nuevas aplicaciones, (Robledo, 2013). Es importante mencionar que en el lenguaje cotidiano, se habla de "investigación" para hacer alusión a la I+D, la investigación no sólo tiene que ver con la generación de nuevo conocimiento, sino también con la aplicación del conocimiento existente para desarrollar nuevos productos y procesos.

Por su parte Jaimes, Ramírez, Vargas y Carrillo, (2011), indica que las actividades de $\mathrm{I}+\mathrm{D}$ en las universidades privadas están relacionadas con la estrategia competitiva de la institución, con su capacidad de liderazgo y nuevas maneras de hacer ciencia. Se debe tener presente otras tecnologías que se desarrollan en paralelo a las actividades de I+D, como las tecnologías de información, transporte, automatización de la oficina, gestión logística, entre otras.

En este sentido, El Kadi y De Pelekais (2015) sugieren que la innovación tecnológica es un proceso orientado a la creación, modificación de las características de un producto/servicio, para introducirlo en el mercado buscando su aceptación por parte de los usuarios y clientes potenciales. Un aspecto esencial de la innovación es su aplicación exitosa de forma comercial, es necesario darlo a conocer y difundirlo para que la gente pueda disfrutar de ello, por tal motivo se requiere de una comunicación organizacional.

La acción de la comunicación para la efectividad de gestión en las organizaciones, ha experimentado la inclusión de esta variable en las políticas o filosofía del proceso de cambio institucional, siendo esta una herramienta hoy día para 
integrar su público la cultura corporativa y armonizar las líneas de mando a seguir, minimizando los conflictos que se pudieran presentar entre estos, considerando la retroalimentación, un indicador de éxito en los espacios de trabajo donde se genere, (Ramírez y Hugueth, 2017).

La innovación tecnológica tiene una mayor aplicación estratégica que operativa, ejecuta iniciativas de desarrollo tecnológico, diseño de productos y procesos. Busca alternativas que proporcionen condiciones de uso mucho más efectivas, consiste en el mejoramiento de productos, servicios y procesos, dentro de líneas existentes, introduciendo modificaciones durante su producción y estancia en el mercado.

En este mismo orden de ideas, Ramírez y Ampudia (2018), manifiesta que innovar contempla introducir nuevas combinaciones entre factores de la producción para generar un producto mejor, nuevo o diferente, que impacte positivamente en el mercado y ambiente, con beneficios económicos como sociales. La tecnología es un instrumento potenciador que aumenta las oportunidades de los países en vías de desarrollo a través del conocimiento. Combinación de información, contexto y experiencia, buscando alcanzar los objetivos del negocio como medio de obtener y administrar el conocimiento que la empresa requiere para ser competitiva.

Por su parte, Jones (2008), afirma que la innovación tecnológica está dada por el proceso organizacional para desarrollar nuevos bienes, servicios, sistemas de producción y operación, respondiendo a las necesidades de sus clientes. Los líderes de la innovación y el desarrollo de nuevos productos son emprendedores internos, es decir, colaboradores que identifican oportunidades para las mejoras cuánticas de sus productos y son responsables de administrar el proceso de desarrollo para la gestión tecnológica.

La responsabilidad del avance tecnológico recae sobre todos los involucrados en las actividades de investigación y desarrollo, quienes de una manera directa deben abordar el estudio de las necesidades de los clientes; las innovaciones implican un 
cambio en la tecnología de producción, estructuras, sistemas administrativos, planes o programas para los miembros de la institución.

Se trata de adquirir tecnologías para administrarlas debidamente, capacidad para aplicarlas y adaptarlas, en beneficio de la comunidad universitaria, también se requiere producirla o crearla. Para ello, es indispensable, tener un acercamiento a otras disciplinas que soportan y complementan el quehacer de las organizaciones universitarias, como punto de apoyo a la evolución del conocimiento, lo administrativo y académico, (Maya, 2012).

Producción y administración estratégica: es un proceso continuado, reiterativo y transfuncional, dirigido a mantener a una organización en su conjunto acoplada, de manera apropiada con el ambiente en el que se desenvuelve. Según Maya (2012), esta definición promueve la: elaboración de un análisis ambiental, fijación de una dirección organizativa, formulación de la estrategia de la organización, ejecución de esta estrategia y ejercicio del control estratégico.

Para Onoyama y otros (2017), la producción y administración estratégica, es una herramienta de planeación y control de la productividad, siendo los insumos requeridos para generar una unidad de producto, contribuyendo significativamente al logro de la efectividad operacional, permitiendo retroalimentar las decisiones, en los resultados de los procesos.

Por su parte, Wu y otros (2018), manifiestan que la producción y administración estratégica, es la formulación, ejecución y evaluación de acciones que permiten que una organización logre sus objetivos. La formulación de estrategias incluye la identificación de las debilidades y fortalezas internas de una organización, determinación de las amenazas y oportunidades externas, establecimiento de misiones de la industria, fijación de los objetivos, desarrollo de las estrategias, alternativas, análisis de dichas alternativas y decisión de cuales escoger.

Utilidad adecuada de la tecnología por el capital social: actualmente la sociedad ha sido protagonistas de la era tecnológica, ocupando espacios en el quehacer diario del talento humano, influenciado significativamente en las vida 
Reynier Ramírez; Giovanni Royero y Omar El Kadi

Telos Vol. 21, No. 1 (2019). 10-32

laboral y personal de cada colaborador, repercutiendo en las organizaciones y sus indicadores de logro. Por tal motivo estas instituciones encargadas de garantizar la consolidación de los objetivos propuestos, ven en la utilidad adecuada de la tecnología por el capital social, una oportunidad para el éxito.

De acuerdo a Wu y otros (2018), la utilidad adecuada de la tecnología por el capital social, se define como el uso adecuado de los recursos tecnológicos en productos, servicios, procesos y áreas estructurales-funcionales, que posibilitan solventar algún problema de la sociedad, ser humano o ambiental. Al mismo tiempo, Onoyama y otros (2017), exponen que esta, se orienta a un mejoramiento de las posibilidades de empleo, competitividad y justicia social tecnológica, repercutiendo en el aprovechamiento de cualquier recurso técnico que garantice la ágil respuesta ante un requerimiento.

Del mismo modo Aguilar (2017), la conceptualiza, como la utilidad de la tecnología asertivamente, facilita la adaptación al ambiente y satisfacción de las necesidades esenciales y deseos de la humanidad. Para Jaimes y otros (2011), es el uso y reconocimiento a cualquier tipo de tecnología, con responsabilidad, para generar resultados efectivos, que garanticen el cumplimiento de los objetivos institucionales, de acuerdo a un proceso sistémico y dinámico.

Ante los aportes de las dimensiones contempladas en la gestión tecnológica, García y Mulero (2007), manifiesta que el factor clave de éxito contempla la eficiencia y rendimiento, como cambio contable de la organización, la denominada gestión tecnológica, es una herramienta con aspectos cualitativos (competencias) y cuánticos (recursos económicos - tecnológicos), en el desarrollo de las actividades de la institución, proporcionando ventajas competitivas.

Al revisar conceptualmente la gestión tecnológica, sus elementos permiten a las universidades privadas, alcanzar los objetivos trazados, distinguiendo a las organizaciones educativas de la competencia, haciéndola única, de acuerdo a su naturaleza, considerándose esta como factor clave de éxito. Se hace necesario resaltar, 
que, al identificar los indicadores de la gestión tecnológica, la academia como organización, podrá saber qué factores claves de éxitos los hace diferentes ante el mercado, para ello deben mirar hacia adentro del negocio, saber cuáles son los procesos o características distintivos de su producto o servicio y cuáles son los que debe dominar a plenitud para crear la ventaja competitiva.

\section{Metodología}

El objetivo de las investigaciones descriptivas es detallar situaciones, es decir, cómo se manifiesta determinado fenómeno, mediante sus propiedades, característica o aspectos. Hurtado (2010), reseña que las investigaciones descriptivas plantean conocer grupos iguales o semejantes de fenómenos, utilizando juicios sistemáticos, colocando de manifiesto su comportamiento, descifrando los hechos a partir de un criterio o modelo teórico definido previamente. Dada sus características, esta investigación se considera de tipo descriptiva, describiendo la gestión tecnológica como factor clave de éxito en las universidades privadas.

El diseño es un método específico que permite a la investigación adaptarse a sus particularidades, indicando las técnicas utilizadas para recolectar y analizar datos. En este caso, el diseño de la investigación es no experimental, según Hernández, Fernández y Baptista (2014), refiere a aquellas donde se observa situaciones, fenómenos y como se dan en su contexto natural para luego analizarlos.

El estudio se ubicó en un diseño transeccional, ya que los datos se recolectaron en un momento y tiempo único. De Pelekais y otros (2015), establece que el diseño transeccional recolecta datos en un momento dado, tiempo único, su objetivo es describir la variable y analizar su incidencia o correlación en un momento específico.

Al respecto, Hurtado (2010), manifiesta que en las investigaciones de campo, los datos de interés se recolectan directamente de la realidad. Ello permite el conocimiento a fondo del problema por parte delos investigadores. Por tal motivo, los datos se recogen directamente de la realidad, garantizando las verdaderas condiciones en que se han obtenido los datos. La investigación se considera con un diseño 
transeccional, los datos se recolectaron en un momento y tiempo; y de campo puesto que se recogieron los datos directamente de la realidad, de fuente fidedigna.

Para Hernández y otros (2014), la población o universo es el conjunto de individuos que tienen propiedades similares, en este caso, cuatro (4) universidades privadas, del municipio Maracaibo, registradas ante el Ministerio del Poder Popular para Educación Universitaria Ciencia, Tecnología (MPPEUCT) de Venezuela, de acuerdo a su naturaleza y propiedad jurídica.

Los criterios de selección de la población sujeto de estudio, responden por su similitud en la estructura organizacional, registradas en el MPPEUCT, utilización de plataformas y herramientas virtuales, cantidad de colaboradores, y naturaleza organizacional; elementos significativos para valorar la gestión tecnológica de estas. Los informantes se caracterizan por ser profesionales: jefes de servicios web, redes, servidores, servicios técnicos, plataformas operacionales y operadores técnicos, siendo cincuenta y cinco (55) personas, (tabla2).

\section{Tabla 2}

\section{Población de las universidades privadas}

\begin{tabular}{|c|c|c|}
\hline $\mathbf{N}^{\circ}$ & Universidades Privadas & Informantes \\
\hline 1 & Universidad Rafael Belloso Chacín. & 18 \\
\hline 2 & Universidad Rafael Urdaneta. & 14 \\
\hline 3 & Universidad José Gregorio Hernández. & 13 \\
\hline \multirow[t]{2}{*}{4} & Universidad Cecilio Acosta. & 10 \\
\hline & Total & 55 \\
\hline
\end{tabular}

Fuente: elaboración propia.

Las técnicas son los medios para recolectar la información, oral o escrita, que recopila directamente el investigador a través de relatos, escritos u otros, transmitidos por los participantes en un acontecimiento, generalmente compuesta por encuestas, cuestionarios, entrevistas, sondeos entre otras, (De Pelekais, El Kadi, Seijo y Neuman, 2015). 
Se utilizó la encuesta como técnica de recolección de datos, y como instrumento el cuestionario que según Hernández y otros (2014) es el conjunto de preguntas respecto a una o más variables a medir. El instrumento se estructuró con la escala tipo Lickert, con cinco (5) alternativas de respuestas: siempre, casi siempre, algunas veces, casi nunca y nunca, conformado por doce (12) ítems, (tabla 3).

Tabla 3

Valores cualitativos y cuantitativos de las alternativas de respuestas

\begin{tabular}{cc}
\hline Valores cualitativos & Valores cuantitativos \\
\hline Siempre & 5 \\
Casi siempre & 4 \\
Algunas veces & 3 \\
Casi nunca & 2 \\
Nunca & 1 \\
\hline
\end{tabular}

Fuente: elaboración propia.

Una vez elaborado el instrumento, el mismo se somete a la validez y confiabilidad. Según Hernández y otros (2014), definen la validez como el grado en el que un instrumento mide la variable. La validez del cuestionario, estuvo a cargo de diez (10) expertos en el área de interés, considerando la redacción, objetividad, relación de los objetivos objeto de estudio con el instrumento, variables, dimensiones e indicaciones. De acuerdo con Hernández y otros (2014), para medir la confiabilidad se utiliza un coeficiente de la formula Alpha Cronbach obteniendo un rtt: 0.80 de resultado, siendo este altamente confiable, para ser aplicado.

Para la realización de este estudio, se tabularon los datos con una matriz de doble entrada en la cual el eje (X)representa los ítems y el eje (Y) está representado por los sujetos, a fin de alcanzar los porcentajes, obteniendo los resultados de forma inmediata. Aplicando posteriormente la estadística descriptiva, mediante el análisis frecuencial y porcentual, de acuerdo a los valores categóricos de interpretación del promedio del baremo, (tabla 4). 
Reynier Ramírez; Giovanni Royero y Omar El Kadi

Telos Vol. 21, No. 1 (2019). 10-32

Tabla 4

Valores categóricos de interpretación del promedio del baremo

\begin{tabular}{cccc}
\hline Alternativas & Valor & Rangos & Categoría \\
\hline Siempre. & 5 & $4,23-5,00$ & Muy alto cumplimiento. \\
Casi siempre. & 4 & $3,42-4,22$ & Alto cumplimiento. \\
Algunas veces. & 3 & $2,61-3,41$ & Mediano cumplimiento. \\
Casi nunca. & 2 & $1,81-2,60$ & Bajo cumplimiento. \\
Nunca. & 1 & $1,00-1,80$ & Muy bajo cumplimiento. \\
\hline
\end{tabular}

Fuente: elaboración propia.

Para Tamayo y Tamayo (2012), indican que el análisis de resultados es el convertir los fenómenos observados en datos científicos, para obtener conclusiones. La información recolectada se agrupo utilizando la distribución de frecuencias absoluta $(\mathrm{Fa})$ y relativa $(\mathrm{Fr})$ para cada uno de los ítems, así como las tendencias, evidenciando el comportamiento de las dimensiones del sistema de la variable.

\section{RESULTADOS OBTENIDOS}

Tabla 5

Gestión tecnológica en las universidades privadas del municipio Maracaibo

\begin{tabular}{|c|c|c|c|c|c|c|c|c|c|c|c|c|c|c|}
\hline \multicolumn{2}{|c|}{$\begin{array}{c}\text { Alternativas de } \\
\text { Respuesta }\end{array}$} & \multicolumn{3}{|c|}{$\mathbf{S}$} & \multicolumn{2}{|l|}{ CS } & \multicolumn{2}{|l|}{ AV } & \multicolumn{2}{|l|}{$\mathrm{CN}$} & \multirow[t]{2}{*}{$\mathbf{N}$} & \multicolumn{2}{|c|}{ TOTAL } & \multirow{3}{*}{$\begin{array}{c}\text { Promedi } \\
\text { o }\end{array}$} \\
\hline & & $\mathbf{F}$ & & $\mathbf{F}$ & & $\mathbf{F}$ & & $\mathbf{F}$ & & $\mathbf{F}$ & & $\mathbf{F}$ & & \\
\hline Indicadores & Ítems & $\mathbf{a}$ & Fr & $\mathbf{a}$ & $\mathbf{F r}$ & $\mathbf{a}$ & $\mathbf{F r}$ & $\mathbf{a}$ & $\mathbf{F r}$ & $\mathbf{a}$ & $\mathbf{F r}$ & $\mathbf{a}$ & $\mathbf{F r}$ & \\
\hline $\begin{array}{c}\text { Actividades } \\
\text { de I+D. }\end{array}$ & $1-3$ & 8 & 14,55 & 19 & 34,55 & 14 & 25,45 & 7 & 12,73 & 7 & 12,73 & 55 & 100 & 2,05 \\
\hline $\begin{array}{l}\text { Innovación } \\
\text { tecnológica. }\end{array}$ & 4-6. & 7 & 12,73 & 13 & 23,64 & 22 & 40,00 & 7 & 12,73 & 6 & 10,91 & 55 & 100 & 2,8 \\
\hline $\begin{array}{l}\text { Producción y } \\
\text { administració }\end{array}$ & $7-9$. & 9 & 16,36 & 10 & 18,18 & 21 & 38,18 & 8 & 14,55 & 7 & 12,73 & 55 & 100 & 3,1 \\
\hline
\end{tabular}




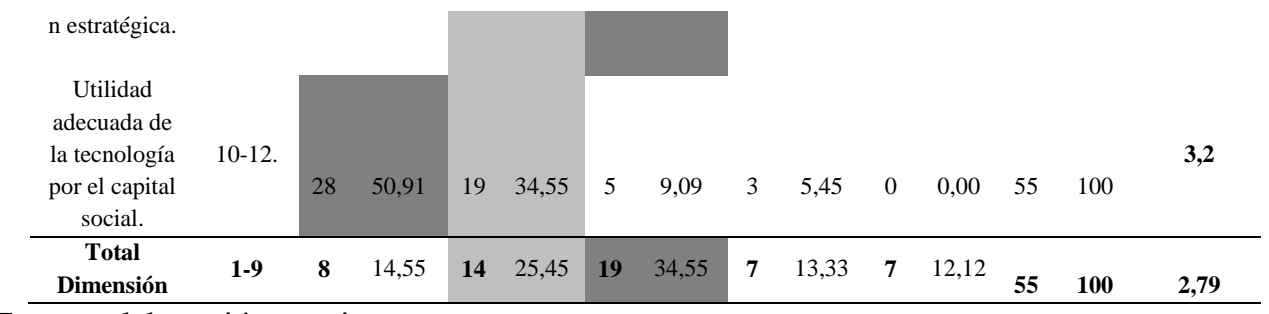

Fuente: elaboración propia.

Al describir la gestión tecnológica como factor clave de éxito en las universidades privadas del municipio Maracaibo, se percibe un mediano cumplimiento en la aplicabilidad de las actividades de I+D, innovación tecnológica, producción y administración estratégica, y utilidad adecuada de la tecnología por el capital social, representada con una media de 2,79. De acuerdo al total de la dimensión, el $34.55 \%$ de los encuestaron emitieron que a veces este proceso se ejecuta, mostrando una neutralidad.

Mientras que un $25,45 \%$ expuso que casi siempre y un14,55\% que siempre, pronunciándose positivamente, favoreciendo la aplicabilidad de la gestión tecnológica en las universidades privadas del municipio Maracaibo, la cual surge cómo una estrategia de gestión, en respuesta a los desafíos impuestos por la globalización de la economía, avances tecnológicos, expansión del uso de nuevas herramientas en información y comunicaciones.

Resultados que obedecen por las actividades de I+D y utilidad adecuada de la tecnología por el capital social, encaminados a fortalecer el desarrollo investigativo, originando una comunidad de práctica, aprendizaje, saber, conocimiento y trasformación, utilizando adecuadamente los recursos tecnológicos para tal fin, posibilitando la construcción y permanencia de los grupos de investigación, centros de desarrollo tecnológicos y en redes.

La gestión tecnológica en las universidades privadas, se vieron orientadas por la innovación, tecnología, flexibilidad productiva y calidad, seleccionándose estos como factores de competitividad, al mismo tiempo transferencia de conocimiento, permitiendo el acceso, modelar estrategias pedagógicas y transformación del nuevo 
conocimiento, aprovechado en el diseño de estrategias de formación, adaptado a las necesidades, realidades, desafíos y cambios del entorno, con éticas e impacto social.

Para Ramírez y Ampudia (2018), hoy en día las empresas juegan un papel vital dentro del entorno, éstas satisfacen tanto las necesidades fisiológicas, psicológicas y de autorrealización social, como los modelos de desarrollo tecnológico y económico de los países. De esta manera, las organizaciones a medida que van apareciendo innovaciones tangibles e intangibles, éstas van cambiando sus estrategias comerciales, así como, su forma de trabajo ya sea interno o externo.

Por otra parte, de los informantes un $13,33 \%$ expuso de que casi nunca y un $12,12 \%$ que nunca se percibe la gestión tecnológica en las universidades privadas, mostrando una tendencia negativa, asumiendo que se desarrollan actividades enfocadas al mantenimiento tecnológico, involucrados en acciones de comercialización y diseño, reflejado en procesos netamente operativos. Al considerar estos resultados, apoya la teoría de Hernández y otros (2017), quienes exponen que la gestión tecnológica, orientado por las actividades de $\mathrm{I}+\mathrm{D}$, e innovación, sólo pueden darse con el dominio de conocimientos, habilidades mediante destrezas asociados a esos procesos que constituyen las capacidades tecnológicas.

De acuerdo a la información expuesta, la gestión tecnológica en las universidades privadas, se concibe como un proceso sistémico administrativo de las actividades de desarrollo tecnológico, vinculando la investigación-organizaciónsociedad, con un enfoque relacional, integrando el proceso de cambio tecnológico con los aspectos estratégicos y operativos del control y la toma de decisiones de las instituciones.

En síntesis, los datos presentados permiten demostrar que la gestión tecnológica, podría considerarse como una variable para establecer sus indicadores y ser un factor clave de éxito en las universidades privadas, vinculando el sector productivo y de la investigación/desarrollo, en el proceso de innovación tecnológica, 
originando conocimientos y prácticas relacionadas con la creación, desarrollo, transferencia y uso de la tecnología.

Los aportes conceptuales de Medellín (2010), Mendoza y otros, (2014), Hernández y otros (2017), Onoyama y otros (2017), y Wu y otros (2018), sustentan lo antes manifiesto, por tal motivo se es necesario fortalecer la gestión tecnológica, a fin de utilizar indicadores pertinentes que garanticen la consolidación de los objetivos de las organizaciones educativas. Al mismo tiempo García y Mulero (2007), expone que la gestión tecnológica, es una herramienta con aspectos cualitativos y cuánticos, en el desarrollo de las actividades de la institución, proporcionando ventajas competitivas, garante del éxito organizacional.

\section{Consideraciones finales}

Por la naturaleza jurídica y razón social de las universidades privadas, su gestión tecnológica se orienta por las actividades de I+D, innovación tecnológica, producción y administración estratégica, con la utilidad adecuada de la tecnología por el capital social. Los hallazgos invitan a diseñar estrategias para la adecuada gestión sobre la variable de estudio.

Las actividades de $\mathrm{I}+\mathrm{D}$, se vieron orientadas a fortalecer y optimizar sus procesos internos y externos, y consolidar la relación universidad - empresa por medio de actividades de transferencia tecnológica. Una fortaleza de estas iniciativas utilizadas por las universidades, fue el respaldo de los organismos administrativos de estas y por tanto se han convertido en políticas que enmarcan el desarrollo de la academia e investigación, facilitando la inserción de estas prácticas en la cultura universitaria.

En cuanto a la innovación tecnológica, se observó el desarrollo de los fundamentos y modelos de gestión tecnológica en la optimización de los procesos administrativos y aplicaciones de soporte a los procesos misionales académicos, como enlaces entre instituciones. Dada la experiencia de las instituciones de educación universitaria en el área de gestión tecnológica, la aplicación de este conocimiento a sus procesos internos constituye una línea de investigación, desarrollándolos con 
proyección y opciones de crecimiento para el quehacer universitario, con un enfoque innovador.

Se evidenció que la gestión tecnológica en la población sujeto de estudio, intégrala organización académica con el mercado, tomando como norte la adaptabilidad al cambio y mejora continua, innovación tecnológica y gestión administrativa. Originado, avances sustanciales sobre las herramientas tecnológicas que poseen las universidades, su utilidad e impacto socio técnico, que corresponden con las exigencias del sistema o entorno; promoviendo la inversión en actividades de investigación y desarrollo de una cultura de conocimiento, posibilitando la innovación constante con enfoque en la productividad intelectual de alto nivel e impacto científico.

En las universidades privadas, se puedo verificar que incluyen en sus principios corporativos la variable gestión tecnológica, garantizando desde su filosofía organizacional el desempeño de sus servicios, aumentando su competitividad y crecimiento como institución en el mercado. Invirtiendo económicamente en la implementación de innovaciones, que se ajusten a su perfil, contribuyendo al crecimiento de la organización, sus innovaciones se enfocan en simplificar los procesos operativos y administrativos, garantes de los indicadores de calidad total.

Por tal motivo, se puede decir que la gestión tecnológica de las universidades privadas, constituyen un indicador de logro necesario dentro de la gerencia, utilizando adecuadamente los recursos tecnológicos por el capital social, para la trasferencia y espacios colaborativos de conocimiento, originando comunidades de práctica, aprendizaje, saber, conocimiento y trasformación.

Estos hallazgos, influyen positivamente en el desarrollo de las universidades privadas, favoreciendo el intercambio de experiencia, local, regional, nacional e internacional, disminuyendo las barreras y distancias geográficas, enriqueciendo el aprendizaje individual y colectivo en los seres humanos. La gestión tecnológica 
universitaria, demanda de la alfabetización tecnológica, dominio de una diversidad de competencias para la sociedad del conocimiento.

A pesar que los resultados, obedecen a un mediano cumplimento de la gestión tecnológica en las universidades privadas del municipio Maracaibo, dichas universidades permanecen en un mercado competitivo cada vez más global, en entornos cambiantes y dinamizadores, poseen convenios con las redes empresariales, cadenas productivas, clúster y stakeholders, han aumentado el número de participantes formados en diferentes niveles de formación, creado enlaces de grupos, semilleros y centros de investigación; gestiones que se podrían aprovechar para ser competitivos en el mercado.

La gestión tecnológica en las universidades privadas, posibilita que las instituciones gestionen conocimiento, permitiendo el acceso, modelar estrategias pedagógicas y transformación del nuevo conocimiento, adaptado a las realidades y desafíos del entorno, con prácticas éticas e impacto social, para la conformación de comunidades de práctica, aprendizaje, saber, conocimiento y transformación.

\section{Referencias bibliográficas}

Aguilar, Marly. (2017). Indicadores de ciencia, tecnología e innovación en Venezuela y su impacto en el desarrollo de políticas públicas. Revista TELOS. Volumen 19, $\mathrm{N}^{\circ}$ 1. Venezuela. (Pp. 119 - 146).

De Pelekais, Cira, El Kadi, Omar, Seijo, Cristina y Neuman Noel. (2015). El ABC de

la Investigación. Pauta Pedagógica. Ediciones Astro Data. Venezuela.

El Kadi Omar y De Pelekais Cira. (2015). E - Comerce, E - Business, E - Logistics y su Aplicación en la Logística Empresarial. Revista Virtual Estudiantil del Programa de Derecho (LEGEM). Volumen 2, N². Colombia. (Pp.63-71).

García Teresa y Mulero Eva. (2007). Medida de los factores claves de éxito de la I+D:

el constructo y sus dimensiones. Revista Cuadernos de Economía y

Dirección de la Empresa. Volumen 18, ’332. España. (Pp.15-47).

Hernández Roberto, Fernández Carlos y Baptista María. (2014). Metodología de la Investigación (sesta edición). Editorial McGraw-Hill Education. México.

Hernández Hugo, Cardona Diego y Del Rio Jorge. (2017). Direccionamiento Estratégico: Proyección de la Innovación Tecnológica y Gestión 
Administrativa en las Pequeñas Empresas. Revista Información Tecnológica.

Volumen 28, $\mathrm{N}^{\circ}$ 5. Chile. (Pp.15-22).

Hurtado de Barrera, Jacqueline. (2010). Metodología de la Investigación Holística

Guía para la compresión holística de la ciencia (cuarta edición). Quirón Ediciones S.A. Colombia.

Jaimes Fuentes Margy, Ramírez Prada Dorys, Vargas Ana María y Carrillo Caicedo Gilberto. (2011). Gestión tecnológica: conceptos y casos de aplicación. Revista Gerencia Tecnológica Informática (GTI). Volumen 10, $\mathrm{N}^{\circ} 26$. Colombia. (Pp. 43 - 54).

Jones, Gareth. (2008). Teoría organizacional. Diseño y cambio de las organizaciones (quinta edición). Editorial Pearson Prentice Hall. México.

Maya Pabón, Raúl. (2012). Gestión tecnológica como componente de la administración estratégica en las organizaciones universitarias. Revista Escenarios. Volumen 10, $\mathrm{N}^{\circ}$ 2. Colombia. (Pp. 111-119).

Medellín Cabrera, Enrique. (2010). Gestión tecnológica en empresas innovadoras mexicanas. RAI - Revista de Administração e Inovação.Volumen 7, $\mathrm{N}^{\circ} 3$. Brasil. (Pp. 58-78).

Mendoza León, Jorge y Valenzuela Valenzuela, Alejandro. (2014). Aprendizaje, innovación y gestión tecnológica en la pequeña empresa Un estudio de las industrias metalmecánica y de tecnologías de información en Sonora. Revista Contaduría y Administración. Volumen 59, º 4. México. (Pp. 253-284).

Onoyama Mori Silvia, Machado Cruz Eduardo, Inoue Nagata Alice y Freitas Simões Jonathan. (2017). Technology Roadmapping, a method to support technology management. RevistaGestao\&tecnologia-journal of management and technology. Volumen 17, $\mathrm{N}^{\circ}$ 2. Brasil. (Pp. 233-251).

Ramírez Molina, Reynier y Hugueth, Alfredo. (2017). Modelo de comunicación productiva para las organizaciones de salud pública en Venezuela. Revista Opción. Volumen 33, No 83. Venezuela. (Pp. 305-335).

Ramírez Molina, Reynier y Ampudia Sjogreen, Daniel. (2018). Factores de Competitividad Empresarial en el Sector Comercial. Revista Electrónica de Ciencia y Tecnología del Instituto Universitario de Tecnología de Maracaibo (RECITIUTM). Volumen 4, $\mathrm{N}^{\circ}$ 1. Venezuela. (Pp.16-32).

Robledo Velásquez, Jorge. (2013). Introducción a la gestión tecnológica (segunda edición). Editorial Universidad Nacional de Colombia. Colombia.

Tamayo y Tamayo, Mario. (2012). Técnicas de la Investigación (tercera edición). Editorial Panapo S. A. España. 
Gestión tecnológica como factor clave de éxito en universidades privadas

Wu Weiwei, Liu Yexin y Chin Tachia. (2018). The effect of technology management capability on new product development in China's service-oriented manufacturing firms: a social capital perspective. Revista Asia Pacific Business Review. Volumen 24, N 2. United Kingdom. (Pp. 212-232). 